\title{
Infective factors and PCT/CRP levels in 112 emergency cases of respiratory tract infection
}

\begin{abstract}
Objectives: To analyze the correlation between (1) the clinical features of respiratory tract infection (RTI) and a poor prognosis in emergency cases and common pathogenic microorganisms and procalcitonin/C-reactive protein (PCT/CRP) in emergency RTIs.
\end{abstract}

Methods: A total of 112 patients were enrolled. The clinical characteristics of RTIs were correlated with common microbes. Patients were grouped based in whether they survived (good prognosis) or died (poor prognosis).

Results: (1) Patients with RTIs were mainly elderly, and the poor prognosis group was on average $\sim 10$ years older than the good prognosis group ( $P=0.0073$ ). (2) No difference in the type of infectious pathogens and mixed infections between the poor prognosis group and the good prognosis group. (3) No significant difference in PCT and CRP between the two groups and the findings suggested that an abnormally high $\mathrm{PCT}$ indicates poor outcomes in patients with emergency RTIs.

Conclusion: (1) Acute RTIs in patients with acute onset and severe illness is generally due to poor immunity. Therefore, in the course of routine anti-bacterial and viral treatment, the basic disease and age of the patient should be taken into consideration. (2) The levels of PCT and CRP may provide guidance for under-diagnosis of viral infections.

Keywords: pulmonary infection, emergency medicine, bacterial infection, viral infection, prognosis
Volume 3 Issue I - 2018

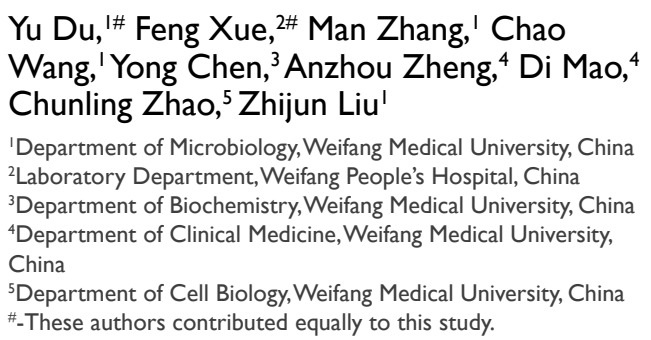

Correspondence: Zhijun Liu, Department of Microbiology, Weifang Medical University, 7166 Bao Tong West Street, Weifang, Shandong 261053, China, Email nluqman2307@gmail.com

Received: February 08, 2018 | Published: February 27, 2018
Abbreviations: RTI, respiratory tract infection; PCT, procalcitonin; CRP, C-reactive protein, SARS, severe acute respiratory syndrome; BUN, blood urea nitrogen.

\section{Background}

Respiratory tract infections (RTIs) are inflammatory diseases caused by pathogenic microorganisms. ${ }^{1}$ In the early stage of infection, the pathogenic factors are usually viruses, and bacterial infections often occur secondary to viral infection. ${ }^{2,3}$ Respiratory viral infections continue to attack human populations, as in the severe acute respiratory syndrome (SARS) epidemic in Hong Kong in 1997 and the H1N1 flu pandemic in 2009. ${ }^{4}$ Similarly, bacterial infections are also a cause of community-acquired respiratory infections. For bacterial infections, we have more antibiotics to choose from, but effective antiviral drugs for respiratory virus infections are not available. ${ }^{5}$ The onset of emergency RTI is generally urgent, the condition is serious, and the prognosis is poor. The main manifestation is pneumonia, and community-acquired pneumonia is the main form. ${ }^{6}$ In recent years, most studies on pneumonia in China and elsewhere have mainly focused on the genetic level to find the cause of the disease; while there have been few epidemiological investigation and little research on the prevention and guidance for treatment. ${ }^{7,8}$ Sputum cultures and drug sensitivity tests are important in the treatment of bacterial pneumonia, but little is known about the correlation between the prognosis for infection by common microorganisms. Viral pneumonia is acute and has a high mortality rate. Clinically, there is no corresponding method of pathogen detection, and treatment cannot be targeted. The pathogenic factors of early RTI are usually viruses. When immunity is low, respiratory viruses can invade the body through the respiratory tract mucosa and cause respiratory tract and systemic inflammatory responses. ${ }^{9}$ In this study, a total of 112 patients with emergency RTIs at Weifang People's Hospital from June, 2013 to June, 2017 were analyzed for correlations between common microorganisms and procalcitonin (PCT/CRP), clinical characteristics, and prognosis, so as to guide treatment and prevent community-acquired pneumonia.

\section{Materials and methods}

\section{Instruments and reagents}

The ATB-Expression semi-automatic bacterial identification and mini Vital automatic fluorescence blood culture instruments were from the Bio Merieu, French; the BSC-1500IIA2-X biological safety cabinet was from Jinan Xin Beixi Biotechnology Co., Ltd, Jinan, China; the blood cell analyzer and the CA1500 automatic hematology analyzer were from Sysmex Co., Ltd, Kobe, Japan; the COBAS8000 automatic biochemical analyzer was from Roche Diagnostics Co., Mannheim, Germany; the Pylon automatic immunoassay system was from Xing Tong Medical Technology Co., Ltd, Suzhou, China; the Nephstar Plus specific protein analyzer was from Shenzhen Guo Sai Biotechnology Co., Ltd, Shenzhen, China; the Vital automatic hem dialysis analyzer was from Shanghai Zhi Heng Medical Devices Co., Ltd, Shanghai, China; the Auction Max 403 automatic urine analyzer was from Ai Kelai Medical Electronics Co., Ltd, Shanghai, China; the 
hydro extractor was from Beijing Bai Yang Medical Co., Ltd, Beijing, China. The Gram-positive and Gram-negative bacteria identification cards were from Mei Liai Diagnostics Products Co., Ltd, Shanghai, China); Luo Qin medium was from Shanghai Fan Ke Biotechnology Co., Ltd, Shanghai, China; white blood cell classification hemolysis (Stromatolyser-4DL), white blood cell classification dye solution (Stromatolyser-4DS), dilution (Cell Pack), and sheath fluid (Cell Sheath) were from Sysmex Co., Ltd.; the ALB kit was from Shandong Gaomi Caihong Analysis Instrument Co., Ltd, China; hypersensitive whole blood CRP test reagents were from Shenzhen Guo Sai Biotechnology Co., Ltd, Shenzhen, China; the calcitonin original quantitative detection kit was from Xing Tong Medical Technology Co., Ltd.; Aution Max 4030 test strips were from Ai Kelai To Medical Electronics Co., Ltd, Shanghai, China.

\section{Research participants and groups}

From June, 2013 to June 2017, 112 patients from the Emergency Medicine Department of Weifang People's Hospital of Shandong Province diagnosed with pulmonary infection were selected as the research participants. The patients of all subjects gave written informed consent to the study protocol, which was approved by the Ethics Committee of Weifang People's Hospital (reference number, wfph2013012). According to survival at discharge, the patients were divided into a good prognosis group, who survived, and a poor prognosis group, who died. The pathogenic microorganisms in the groups were compared and analyzed. The average age was $67.96 \pm 16.07$ years ( 5 were $<40$ years old, and 81 were $>60$ years old (Figure 1$)$. In terms of medical history, $47.35 \%$ patients had hypertension, $24.18 \%$ had diabetes, $25.19 \%$ had coronary heart disease, $23.17 \%$ had chronic obstructive pulmonary disease, $14.10 \%$ had cerebrovascular disease, and $1.1 \%$ had lung cancer. There were 48 smokers in the study group (42.86\%), (Figure 2). The diagnoses at admission were type 1 respiratory failure (1 case, $0.89 \%)$, hypostatic pneumonia $(1,0.89 \%)$, bronchiectasia $(2,1.79 \%)$, interstitial pneumonia $(2,1.79 \%)$, fungal pneumonia $(2,1.79 \%)$, severe pneumonia $(7,6.25 \%)$, pulmonary infection $(17,15.18 \%)$, acute onset of chronic obstructive pulmonary disease $(19,16.96 \%)$, and bacterial pneumonia $(57,50.89 \%)$. Only 4 cases $(3.57 \%)$ were diagnosed with viral pneumonia (Figure 3 ).

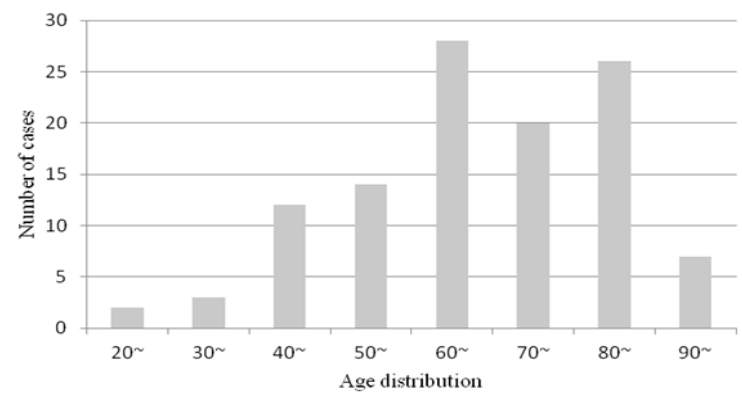

Figure I Age distribution of patients.

\section{Statistical methods}

SPSS16.0 statistical software (SPSS, Inc., Chicago, Illinois) was used to analyze data. Data are expressed as mean \pm standard deviation. The two groups were compared using the independent sample t test or the Mann-Whitney test. A difference at $\mathrm{P}<0.05$ was considered statistically significant.

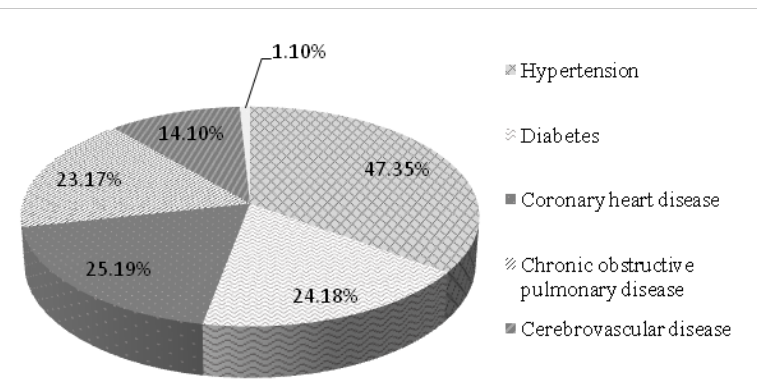

Figure $\mathbf{2}$ History of patients with emergency respiratory tract infection.

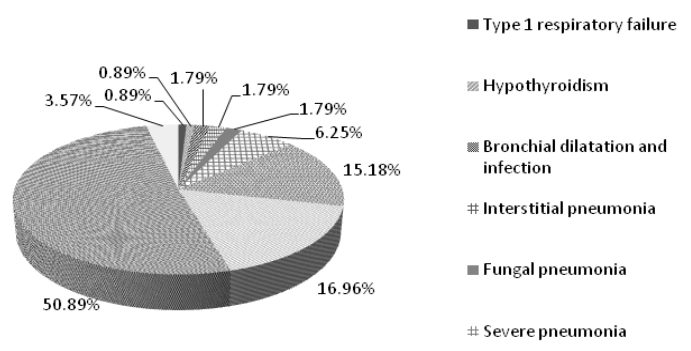

Figure 3 Diagnoses of emergency respiratory tract infection.

\section{Results}

\section{Pathogenic bacteria cultures of sputum from emergency patients with severe respiratory infection}

In this study, a total of 16 species of bacteria were detected in the sputum cultures of patients with severe RTIs, and mixed bacterial infections were commonly found. Among these, the most common bacteria were Streptococcus mitis and Neisseria sicca, which usually occurred together. In addition, more than half of the cultures showed N. sicca, Klebsiella pneumoniae, Acinetobacter baumannii, Candida albicans, Staphylococcus aureus, and Serratia marcescens. $S$. marcescens can become pathogenic when host immunity is dysfunctional, causing lung infection, meningitis, sepsis, or other systemic infections. The specific spectrum of pathogenic bacteria in sputum cultures is shown in (Figure 4).

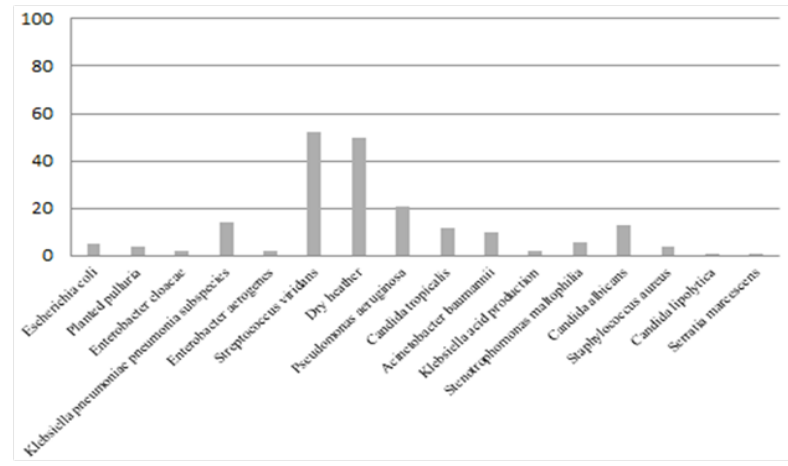

Figure 4 Pathogenic microorganism spectrum in sputum cultures from emergency patients with severe respiratory infection. 


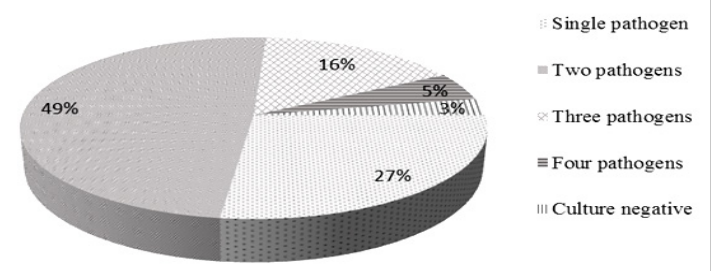

Figure 5 Proportions of multiple infections in sputum cultures from emergency patients with severe respiratory infection.

\section{Combined infection with pathogenic bacteria from sputum of emergency patients with severe respiratory infection}

Combined bacterial infections in patients with severe RTIs are common, so we used sputum to identify multiple infections of the lungs. Our results showed that the infection rate for a single species was $27 \%$, for two, $49 \%$, for three, $16 \%$, and for four, $5 \%$. Among these, the highest proportion was of two pathogenic bacteria, most commonly viridians streptococci combined with $N$. sicca. A possible reason is the prevalence of low immunity in elderly patients with acute RTIs (Figure 5).

\section{Analysis of factors associated with a poor prognosis in patients with severe respiratory tract infection}

\section{Emergency treatment of severe respiratory tract infection in patients with a poor prognosis}

Of the 112 patients enrolled, 35 (46.1\%) died. Comparative analysis showed that the age of the good prognosis group was $65.79 \pm 1.78$ years, while that of the poor prognosis group was $75.48 \pm 2.27$ years $(\mathrm{P}$ $=0.0073)$. Thus, the older the patient with emergency RTI, the higher the fatality rate (Table 1).

Table I Acute respiratory tract infection in patients with different prognosis

\begin{tabular}{llll}
\hline Index & Good prognosis & $\begin{array}{l}\text { Poor } \\
\text { prognosis }\end{array}$ & $\mathbf{P}$ \\
Age $(y)$ & $65.79 \pm 1.782$ & $75.48 \pm 2.275$ & 0.0073 \\
Sex $(F / M)$ & $26 / 62$ & $14-$ Oct & $>0.05$ \\
\hline
\end{tabular}

\section{Routine blood and biochemical indexes in emergency treatment of severe respiratory tract infection}

The number of neutrophils in the good prognosis group was significantly lower than that in the poor prognosis group, suggesting a more serious bacterial infection causing an increase of neutrophils in the latter group. Blood biochemical analysis of lactic acid, aspirate aminotransferase, alanine aminotransferase, gamma-glutamyl transferase, creatinine, blood urea nitrogen (BUN), and lactate dehydrogenate showed no significant differences between the two groups, except for BUN (Table 2) (Table 3). BUN is the major endproduct of protein metabolism, and when the glomerular filtration rate is less than half of normal, the BUN rises rapidly. Since the BUN in the poor prognosis group was higher than that in the improved group, it was presumed to be related to a higher incidence of renal dysfunction in that group.

\section{Levels of procalcitonin (PCT) and C-reactive protein (CRP) at admission of patients with severe respiratory tract infection}

The average PCT in the good prognosis group was $1.682 \pm 0.25$ $\mathrm{mg} / \mathrm{ml}$, and in the poor prognosis group $1.750 \pm 0.19 \mathrm{mg} / \mathrm{ml}$, showing no significant difference. In the CRP test, the value for the good prognosis group was $62.40 \pm 16.84 \mathrm{mg} / \mathrm{l} / \mathrm{h}$ and in the poor prognosis group $20.02 \pm 10.51 \mathrm{mg} / \mathrm{l} / \mathrm{h}$, also showing no significant difference.

Table 2 Routine blood indexes in patients with severe respiratory tract infection

\begin{tabular}{llcc}
\hline Index & Improved Group & Dead Group & $\begin{array}{l}\mathbf{P} \\
\text { value }\end{array}$ \\
\hline $\begin{array}{l}\text { Blood gas } \\
\text { analysis }\end{array}$ & $7.445 \pm 0.008862$ & $7.41 \pm 0.02201$ & 0.09 \\
$\begin{array}{l}\text { White blood } \\
\text { cells }\end{array}$ & $10.15 \pm 0.6532$ & $12.49 \pm 1.720$ & $>0.05$ \\
$\begin{array}{l}\text { Neutrophils } \\
\text { Platelets }\end{array}$ & $8.262 \pm 0.5432$ & $11.05 \pm 1.702$ & 0.0422 \\
\hline
\end{tabular}

Table 3 Biochemical indexes in severe respiratory tract infection

\begin{tabular}{llll}
\hline Index & $\begin{array}{l}\text { Improved } \\
\text { Group }\end{array}$ & Dead Group & P value \\
\hline $\begin{array}{l}\text { Lactic acid } \\
\begin{array}{l}\text { Aspartate } \\
\text { aminotransferase }\end{array}\end{array}$ & $67.72 \pm 21.09$ & $227.7 \pm 183.5$ & $>0.05$ \\
$\begin{array}{l}\text { Alanine } \\
\text { aminotransferase }\end{array}$ & $100.5 \pm 48.37$ & $179.9 \pm 146.5$ & $>0.05$ \\
$\begin{array}{l}\gamma \text {-glutamyl transferase } \\
\text { Blood urea nitrogen }\end{array}$ & $69.99 \pm 9.521$ & $68.91 \pm 15.29$ & $>0.05$ \\
$\begin{array}{l}\text { Creatine } \\
\text { Lactate dehydrogenase }\end{array}$ & $319.8 \pm 23.88$ & $517.6 \pm 210.7$ & $>0.05$ \\
\hline
\end{tabular}

\section{Changes of PCT and CRP indexes in patients with} severe respiratory tract infection

While the PCT and CRP values did not differ between the two groups of patients at admission, those in the good prognosis group returned to the normal level. However, in one poor prognosis case, the patient had a PCT $>100 \mathrm{mg} / \mathrm{ml}$, while another whose PCT was $35.28 \mathrm{mg} / \mathrm{ml}$ at admission, it rose to $>100 \mathrm{mg} / \mathrm{ml} 5$ days later, when the patient died. The patients in the good prognosis group did not have $>20 \mathrm{mg} / \mathrm{ml}$ PCT in the course of treatment. Therefore, this study is consistent with reports that PCT and CRP can be used as an indicator of bacterial infection; in addition, our results suggest an abnormal increase of PCT in patients with RTIs strongly suggests a poor prognosis.

\section{Correlation between poor prognosis and medical history and smoking in emergency intensive respiratory tract infections}

A poor prognosis is often related to underlying diseases, and the severity of RTIs is associated with past hypertension, diabetes, and other diseases. Smoking is particularly associated with RTIs. In 
this regard, we analyzed all the underlying diseases in 112 patients. Found 82 cases improved in the group of patients with hypertension in 35 cases, and 25 cases died in the group of patients, 17 patients with smoking, $\mathrm{P}=0.0389$, statistically, similarly, the complications associated with cerebral infarction case were higher, $\mathrm{P}=0.044$. An analysis of the history of diabetes, smoking, and chronic obstructive pulmonary disease showed no significant difference between the two groups (Table 4). Based on the above, smoking point to a poor prognosis of severe RTIs in the emergency department.

Table 4 Respiratory tract infections and basic diseases/smoking

\begin{tabular}{llll}
\hline Basic diseases/smoking & $\begin{array}{l}\text { Good } \\
\text { prognosis }\end{array}$ & $\begin{array}{l}\text { Poor } \\
\text { prognosis }\end{array}$ & P \\
\hline Hypertension (yes/no) & $35 / 47$ & $17 / 8$ & 0.0389 \\
Diabetes (yes/no) & $15 / 73$ & $8 / 17$ & $>0.05$ \\
Smoking (yes/no) & $37 / 51$ & $14 / 14$ & $>0.05$ \\
$\begin{array}{l}\text { Cerebral infarction (yes/no) } \\
\text { Chronic obstructive }\end{array}$ & $20 / 68$ & $11 / 14$ & 0.044 \\
pulmonary disease (yes/no) & $15 / 73$ & $8 / 17$ & $>0.05$ \\
\hline
\end{tabular}

\section{Prognosis of severe respiratory tract infection in emergency department and its correlation with infectious pathogens}

The proportions of multiple infections in the good and poor prognosis groups was higher than that in the single-infection group, and there was no significant difference between the two groups. This shows that the deaths were not due to the variety of pathogenic bacteria or species. Presumably, they are associated with a patient's autoimmune status, i.e., immunocompromised patients among those critically ill with severe TRIs.

\section{Discussion}

The incidence of pneumonia has been increasing year by year. ${ }^{10} \mathrm{At}$ the same time, the overuse of antibiotics has led to the emergence of multidrug-resistant bacteria, which are responsible for the increase in the number of elderly patients affected..$^{11}$ Surveillance is an important way to detect infectious diseases, the most important aspect of which is to conduct laboratory tests on pneumonia cases, identify pathogens, and provide references for treatment, prevention, and control. ${ }^{12-14}$ Therefore, rapid, accurate, and comprehensive laboratory testing methods are the future trend. Patients in the emergency intensive care unit with serious RTIs had lowered immunity. ${ }^{15}$ Analysis of patients with a good versus a bad outcome showed that the latter was $\sim 10$ years older than the former, while there was no difference in the type of infection-causing bacteria between the two groups. CRP has been used to evaluate the severity of bacterial infection and prognosis in clinical and research work. ${ }^{16}$ The sensitivity of CRP is very high, only found in trace amounts in normal human serum, but beginning to rise within a few hours of pulmonary infection. ${ }^{17} \mathrm{PCT}$, one of the precursors of calcitonin, is a more sensitive and specific marker of bacterial infection than CRP. ${ }^{18,19}$ It is believed that the serum PCT in bacterial RTI is significantly higher than that due to other pathogenic microorganisms. Many of the clinical guidelines for bacterial infection include PCT as an important indicator of diagnosis. Most studied is the use of PCT in community-acquired and ventilatorassociated pneumonia. ${ }^{20}$ Studies have shown that PCT can be used for the differential diagnosis of bacterial from non-bacterial infection, ${ }^{18,21}$ and can guide the clinical use of antibiotics. ${ }^{22}$ Conversely, in viral and parasitic infections or autoimmune diseases and other non-infectious diseases, serum PCT levels are not elevated or only increase slightly. Therefore, the serum levels of PCT are used to determine whether the initial infection is bacterial or non-bacterial.

In this study, the serum PCT/CRP in 112 emergency cases of RTI showed no significant differences between the two groups at admission, but both decreased significantly in the poor prognosis group, while they almost returned to the normal levels in the good prognosis group. For example, one patient had a of PCT $35.28 \mathrm{mg} / \mathrm{ml}$ at admission, but 5 days later, the PCT was $>100 \mathrm{mg} / \mathrm{ml}$, and the patient died that day. During the course of treatment, patients in the good prognosis group had PCT values not more than $20 \mathrm{mg} / \mathrm{ml}$. Therefore, an elevated PCT in patients with an emergency RTI strongly suggests a poor prognosis. Viral infection is an important cause of RTIs, mainly in hospitalized infants and children in developed countries, but it is a major cause of death in the developing world. ${ }^{2,23}$ However, among the 112 cases in our study, only 4 were diagnosed with viral pneumonia, probably because of a general assumption of bacterial pneumonia. Many previous studies have seriously underestimated the incidence of pneumonia caused by viral infections, especially by new respiratory viruses, which can cause severe pneumonia with a high fatality rate ${ }^{24-26}$. Taking the 2003 SARS outbreak as an example, the Asian Development Bank estimated that this epidemic cost Hong Kong $\sim \$ 6$ billion. Early detection of outbreaks is the key to preventing the spread of pneumonia, and pathogen monitoring is the primary means of identifying new outbreaks of infectious disease virus, such as the 2013 H7N9 avian influenza virus infection. ${ }^{27}$ However, the widespread lack of diagnostic techniques for respiratory virus's leads to a low diagnostic rate, accompanied by inadequate awareness among clinicians. Nevertheless, with the development of molecular detection technology, the diagnostic rate of viral pneumonia has been increasing steadily, especially considering the outbreaks of new influenza viruses, such as H1N1, the SARS coronavirus, the Middle East respiratory syndrome coronavirus, and the highly pathogenic avian influenza viruses H5N1 and H7N9. ${ }^{28,29}$ Research has shown that in both developed and developing countries, influenza virus, par influenza virus, rhinovirus, respiratory syncytial virus, cytomegalovirus, and bocavirus are still the main causes of pneumonia. ${ }^{30-34}$ Therefore, accurate and rapid identification of pathogens is critical for effective antiviral treatment and for infection control. However, a range of respiratory viruses can trigger similar clinical symptoms, making diagnosis difficult. Thus, not only is rapid diagnostic technology needed in hospitals at all levels, but the ability of clinicians to identify viral pneumonia needs improvement.

In summary, we investigated clinical features of patients with emergency RTIs and the correlation between infection and poor prognosis, especially in elderly patients. Although PCT and CRP monitoring is necessary to determine the type of pathogenic microorganism and provide a basis for the severity and prognosis, in 112 cases of emergency patients with RTIs in this study, only 4 were diagnosed as viral pneumonia, probably because viral pneumonia mostly occurred along with bacterial infection and was often judged to be bacterial pneumonia, due to an inadequate understanding of viral pneumonia. Therefore, multiple pathogen detection technology and rapid screening can significantly improve the clinical treatment, avoid the overuse of antibiotics, and detect common and new outbreaks of TRIs early. 


\section{Conclusion}

Acute RTIs in patients with acute onset and severe illness is generally due to poor immunity. Therefore, in the course of routine anti-bacterial and viral treatment, the basic disease and age of the patient should be taken into consideration. The levels of PCT and CRP may provide guidance for under-diagnosis of viral infections.

\section{Acknowledgement}

This work was funded by grants from the National Natural Science Foundation of China (81471048), the Natural Science Foundation of Shandong Province (ZR2012HL42, ZR2015HM028), the Project of Shandong Province Higher Educational Science and Technology Program (J12LK01), Weifang development program of science and technology (2014WS0021), doctoral Scientific Research Foundation of Weifang Medical University (2017BSQD01) and Students' science and technology innovation project of Weifang Medical Univeristy (KX2017031).

\section{Conflict of interest}

The authors declare no conflicts of interest.

\section{References}

1. Jesenak M, Urbancikova I, Banovcin P. Respiratory Tract Infections and the Role of Biologically Active Polysaccharides in Their Management and Prevention. Nutrients. 2017;9(7).

2. Allie SR, Randall TD. Pulmonary immunity to viruses. Clin Sci (Lond). 2017;131(14):1737-1762.

3. Liu H Hot Topics in Primary Care: Community-Acquired Bacterial Pneumonia: Is There Anything New? J Fam Pract. 2017;66(4):10-15.

4. Hendrickson CM, Matthay MA. Viral pathogens and acute lung injury: investigations inspired by the SARS epidemic and the 2009 H1N1 influenza pandemic. Semin Respir Crit Care Med. 2013;34(4):475-486.

5. Rodrigo Troyano A, Sibila O. The respiratory threat posed by multidrug resistant Gram-negative bacteria. Respirology. 2017;22(7):1288-1299.

6. Lee KY. Pneumonia, Acute Respiratory Distress Syndrome, and Early Immune-Modulator Therapy. Int J Mol Sci. 2017;18(2):e388.

7. Liu J, Pang Z, Wang G, et al. Advanced Role of Neutrophils in Common Respiratory Diseases. J Immunol Res. 2017;e6710278.

8. Li H, Cao B. Pandemic and Avian Influenza A Viruses in Humans: Epidemiology, Virology, Clinical Characteristics, and Treatment Strategy. Clin Chest Med. 2017;38(1):59-70.

9. Tregoning JS, Schwarze J. Respiratory viral infections in infants: causes. clinical symptoms, virology, and immunology. Clin Microbiol Rev. 2010;23(1):74-98.

10. Wunderink RG, Waterer G. Advances in the causes and management of community acquired pneumonia in adults. BMJ. 2017;358:2471.

11. Cilloniz C, Martin Loeches I, Garcia Vidal C, et al. Microbial Etiology of Pneumonia: Epidemiology, Diagnosis and Resistance Patterns. Int J Mol Sci. 2016;17(12):2120

12. Izadnegahdar R, Cohen AL, Klugman KP, et al. Childhood pneumonia in developing countries. Lancet Respir Medicine. 2013;(7):574-584.

13. Fleming DM, Zambon M. Update on influenza and other viral pneumonias. Curr Opin Infect Dis. 2010;14(2):199-204.

14. Jain S, Self WH, Wunderink RG, et al. Community-Acquired Pneumonia Requiring Hospitalization among U.S. Adults. The New England journal of medicine. 2015;373(5):415-427.
15. Arthur LE, Kizor RS, Selim AG, et al. Antibiotics for ventilatorassociated pneumonia. Cochrane Database Syst Rev. 2016;10:e004267.

16. De Oliveira VM, Moraes RB, Stein AT. Accuracy of C-Reactive protein as a bacterial infection marker in critically immunosuppressed patients: A systematic review and meta-analysis. J Crit Care. 2017;42:129-137.

17. Gao L, Liu X, Zhang D, et al. Early diagnosis of bacterial infection in patients with septicopyemia by laboratory analysis of PCT, CRP and IL6. Exp Ther Med. 2017;13 (6):3479-3483.

18. Taylor R, Jones A, Kelly S, et al. A Review of the Value of Procalcitonin as a Marker of Infection. Cureus. 2017;9(4):e1148.

19. Principi N, Esposito S. Biomarkers in Pediatric Community-Acquired Pneumonia. Int J Mol Sci. 2017;18(2):447.

20. Nobre V, Borges I, Nucleo Interdisciplinar De Investigacao Em Medicina I. Prognostic value of procalcitonin in hospitalized patients with lower respiratory tract infections. Rev Bras Ter Intensiva. 2016;28(2):179-189.

21. Masajtis Zagajewska A, Nowicki M. New markers of urinary tract infection. Clin Chim Acta. 2017;471:286-291.

22. Sager R, Kutz A, Mueller B, et al. Procalcitonin-guided diagnosis and antibiotic stewardship revisited. BMC Med. 2017;15(1): 15.

23. Camp JV, Jonsson CB. A Role for Neutrophils in Viral Respiratory Disease. Front Immunol. 2017;8:550.

24. Asai N, Motojima S, Ohkuni Y, et al. Clinical Manifestations and Prognostic Factors of Pneumocystis jirovecii Pneumonia without HIV. Chemotherapy. 2017;62(6):343-349.

25. Premaratna R, Luke N, Perera H, et al. Sporadic cases of adult measles: a research article. BMC Res Notes. 2017;10(1):38.

26. Yu Z, Zeng Z, Zhang J, et al. Fatal Community-acquired Pneumonia in Children Caused by Re-emergent Human Adenovirus 7d Associated with Higher Severity of Illness and Fatality Rate. Sci Rep. 2016;6:e37216.

27. Danqi B, Li Z, Liu Q, et al. H7N9 avian influenza A virus in China: a short report on its circulation, drug resistant mutants and novel antiviral drugs. Expert Rev Anti Infect Ther. 2017;15(8):723-727.

28. Al Mohaissen M. Awareness among a Saudi Arabian university community of Middle East respiratory syndrome coronavirus following an outbreak. East Mediterr Health J. 2017;23(5):351-360.

29. Cheng YH, You SH, Lin YJ, et al. Mathematical modeling of postcoinfection with influenza A virus and Streptococcus pneumoniae, with implications for pneumonia and COPD-risk assessment. Int J Chron Obstruct Pulmon Dis. 2017;12:1973-1988.

30. Russell E, Ison MG. Parainfluenza Virus in the Hospitalized Adult. Clin Infect Dis. 2017;65(9):1570-1576.

31. Yan F, Xiao Y, Li M, et al. Metagenomic Analysis Identified Human Rhinovirus B91 Infection in an Adult Suffering from Severe Pneumonia. Am J Respir Crit Care Med. 2017;195(11):1535-1536.

32. Karademir S, Yoldas T Clinical course of community-acquired respiratory syncytial virus pneumonia in newborns hospitalized in neonatal intensive care unit. Tuberk Toraks. 2017;65(1):64-65.

33. Coclite E, Di Natale C, Nigro G. Congenital and perinatal cytomegalovirus lung infection. J Matern Fetal Neonatal Med. 2013;26(17):1671-1675.

34. Khalfaoui S, Eichhorn V, Karagiannidis C, et al. Lung Infection by Human Bocavirus Induces the Release of Profibrotic Mediator Cytokines In Vivo and In Vitro. PLoS One. 2016;11(1):e0147010. 